\title{
Nucleosynthetic Constraints on the Progenitor of Cassiopeia $\mathrm{A}:{ }^{26} \mathrm{Al}$ and $\mathrm{O}$ isotopes compared to SMOW
}

\section{Carola Ellinger*}

Arizona State University

E-mail: carola.ellingercasu.edu

\section{Patrick A. Young}

Arizona State University

E-mail: pyoung3easu. edu

\section{Chris L. Fryer}

Los Alamos National Laboratory

E-mail: fryerelanl.gov

\begin{abstract}
Our recent 3D simulation of a supernova explosion to constrain the progenitor of Cas $\mathrm{A}$ has enabled us to gain new insight into burning processes at work. Of special interest in this poster are the isotopes ${ }^{26} \mathrm{Al},{ }^{16} \mathrm{O},{ }^{17} \mathrm{O}$, and ${ }^{18} \mathrm{O}$. These isotopes are important in the formation history of our solar system, and help constrain its environment at the time of formation. A favored model is the addition of supernova ejecta to the forming solar system, which presumably changed the isotopic abundances of a range of isotopes (like those mentioned above). In this poster we investigate how the relative abundances of the oxygen isotopes in the solar system is affected by the injection of supernova material, given that this injection produces the expected change in ${ }^{26} \mathrm{Al} /{ }^{27} \mathrm{Al}$. We also investigate whether the bulk composition or specific zones of the supernova are more appropriate, and what the determining factors are. Lastly, we look at differences between $1 \mathrm{D}$ and the 3D simulation in this matter.
\end{abstract}

10th Symposium on Nuclei in the Cosmos

July 27 - August 12008

Mackinac Island, Michigan, USA

\footnotetext{
* Speaker.
} 


\section{The Simulations}

Four progenitor models were used: $40 \mathrm{M}_{\odot}$ and $23 \mathrm{M}_{\odot}$ single stars, and $16 \mathrm{M}_{\odot}$ and $23 \mathrm{M}_{\odot}$ binary stars (Young et al. 2006). The binary stars had their $\mathrm{H}$ envelope removed at the onset of the red giant phase to mimic a common-envelope evolution. The progenitors were produced with the TYCHO stellar evolution code of Young \& Arnett (2005) with the solar abundances of Grevesse \& Sauval (1998). These models were then exploded with different parameters in 1D using a 14 element network to follow energy generation.

In addition to that, the explosion of the simulation 23m-run5 was modeled in 3D. After removal of the $\mathrm{H}$ envelope it evolved as a WNL and WN to a final mass of $6.4 \mathrm{M}_{\odot}$ (Young et al. 2006). After the collapse and launch of the explosion in 1D, the simulation was mapped into the 3D explosion code SNSPH (Fryer et al. 2005). An asymmetry was introduced by increasing the velocities of the particles within 30o of the z-axis by a factor of 6 (high velocity structures), and decreasing the velocity of all other particles by 1.2 to conserve explosion energy.

To accurately calculate the yields of the explosion we turned to a post-process step. The postprocessing was performed with the Burn code of Young \& Fryer (2007) and used a 524-element network.

\section{2. ${ }^{26} \mathrm{Al}$ in 3D}
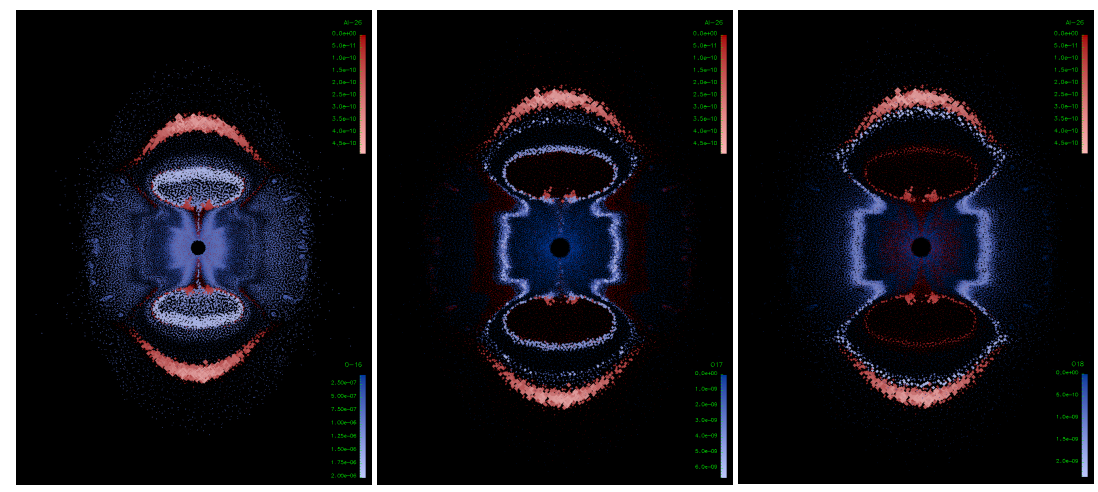

Figure 1: Shown are the masses in $\mathrm{M}_{\odot}$ for ${ }^{26} \mathrm{Al}$ (red), ${ }^{16} \mathrm{O},{ }^{17} \mathrm{O}$, and ${ }^{18} \mathrm{O}$ (each in blue) for the simulation as a slice through the center of the explosion.

${ }^{26} \mathrm{Al}$ is produced in two main regions (see figure 1). The first is at the terminal end of the high velocity structure and contains the highest concentration of ${ }^{26} \mathrm{Al}$. It will be referred to as the Bubble. The second region is in a smaller bubble and particularly a ring around the high velocity structure where the latter emerges into a lower-density region. This will be called the ring. The Bubble is comprised of a region that underwent hydrostatic $\mathrm{C}$ burning in the progenitor, and reached peak-shock temperatures of $\sim 1.5 \cdot 10^{9} \mathrm{~K}$ during the explosion. The material in the ring underwent hydrostatic $\mathrm{Ne}$ burning in the progenitor and explosive $\mathrm{Ne}$ and $\mathrm{C}$ burning during the explosion at temperatures slightly above $2 \cdot 10^{9} \mathrm{~K}$.

${ }^{26} \mathrm{Al}$ is usually quickly destroyed in succeeding reactions. However, due to the rapid expansion of the high velocity structure the density drops rapidly, and a freezeout phase is reached in the 
Bubble. This is the reason for the higher production of ${ }^{26} \mathrm{Al}$ in the sub-explosive $\mathrm{C}$ burning region (Bubble) as opposed to the explosive $\mathrm{C} / \mathrm{Ne}$ burning region (ring) without subsequent destruction. The opposite of that is observed in the 1D simulations.

\section{3. ${ }^{26} \mathrm{Al}$ in $1 \mathrm{D}$}

The peaks in ${ }^{26} \mathrm{Al}$ abundances coincide with temperatures slightly above $2 \cdot 10^{9} \mathrm{~K}$ and at 1.5 . $10^{9} \mathrm{~K}$ in material that has undergone hydrostatic $\mathrm{C}$ burning in the progenitor. The higher of the temperatures is sufficient for explosive $\mathrm{C}$ and Ne burning. These conditions are similar to those reached in the ring in the $3 \mathrm{D}$ simulation. At temperatures $\sim 1.5 \cdot 10^{9} \mathrm{~K}$ there is no major burning stage, but the abundance of free $\mathrm{p}, \mathrm{n}$, and alphas is significantly increased, enhancing production of species by captures of those free particles. This significantly increases the abundance of ${ }^{26} \mathrm{Al}$. This is similar to the conditions that exist in the Bubble. At even higher ( $\mathrm{O}$ burning) temperatures, ${ }^{26} \mathrm{Al}$ is quickly destroyed.

\section{Oxygen}

${ }^{16} \mathrm{O}$ is one of the main burning products of explosive $\mathrm{C} / \mathrm{Ne}$ burning, therefore it is quite abundant in the ring. Some of the free $\mathrm{p}$ and $\mathrm{n}$ produced during this explosive go into producing ${ }^{17} \mathrm{O}$. The temperatures in the Bubble are not sufficient for explosive burning, thus ${ }^{16} \mathrm{O}$ there is mostly leftover from the hydrostatic $\mathrm{C}$ burning in the progenitor. The increased flux of free particles burns some of the ${ }^{16} \mathrm{O}$ there to ${ }^{18} \mathrm{O}$ (via ${ }^{17} \mathrm{O}$ ). The detailed production of ${ }^{18} \mathrm{O}$ is, however, sensitive to the thermodynamic history of the explosion.

\section{Oxygen Isotopes in the Early Solar System}

There is evidence in the form certain unstable isotopes (called short-lived radionuclides) found in meteorites that our solar system was born near a supernova (e.g. Desch \& Ouellette 2005,

\begin{tabular}{|c|c|c|c|c|c|}
\hline \multicolumn{6}{|c|}{ Results } \\
\hline & Bulk & Bubble & Ring & Torus & $\begin{array}{c}\text { Region } \\
\text { outside Bubble }\end{array}$ \\
\hline \multicolumn{6}{|c|}{ "maximum impact" scenario } \\
\hline $\begin{array}{c}\delta^{17} \mathrm{O} \\
\delta^{18} \mathrm{O} \\
\text { Fraction }\end{array}$ & $\begin{array}{l}-106^{0} / 00 \\
+612^{0} / 00\end{array}$ & $\begin{array}{l}-37.3^{0} / 00 \\
-39.8^{0} / 00\end{array}$ & $\begin{array}{l}-223^{0} / 00 \\
+46.2^{0} / 00\end{array}$ & $\begin{array}{c}-87.8^{0} / 00 \\
-14.8^{0} / 00 \\
1 \cdot 10^{-4}\end{array}$ & $\begin{array}{c}-13.4^{0} / 00 \\
-15.8^{0} / 00 \\
1 \cdot 10^{-3}\end{array}$ \\
\hline \multicolumn{6}{|c|}{ "minimum impact" scenario } \\
\hline $\begin{array}{c}\delta^{17} \mathrm{O} \\
\delta^{18} \mathrm{O} \\
\% \text { of } \mathrm{O} \text { in dust }\end{array}$ & $\begin{array}{c}-66.2^{0} / 00 \\
-218^{0} / 00 \\
54 \%\end{array}$ & $\begin{array}{c}-37.3^{0} / 00 \\
-39.8^{0} / 00 \\
100 \%\end{array}$ & $\begin{array}{c}-223^{0} / 00 \\
+46.2^{0} \% \\
100 \%\end{array}$ & $\begin{array}{c}-87.8^{0} / 00 \\
-14.8^{0} / 00 \\
100 \%\end{array}$ & $\begin{array}{c}-38.6^{0} / 00 \\
-38.7^{0} / 00 \\
5.5 \%\end{array}$ \\
\hline
\end{tabular}

Table 1: This table shows our estimated pre-injection oxygen composition. For comparison, first results from the Genesis Mission indicate that the solar (i.e. the presumably true pre-injection) oxygen composition is at $\left(\delta^{17} \mathrm{O}, \delta^{18} \mathrm{O}\right) \sim\left(-60^{0} / 00,-60^{0} / 00\right)$ (S. J. Desch, private communication). 
Tachibana et al. 2006). This supernova is believed to have injected part of its ejecta into the forming solar system, and thus contributed to its inventory of (both stable and unstable) isotopes. The most studied of the unstable isotopes is ${ }^{26} \mathrm{Al}$. Its half life is too short to have been inherited from the material out of which the solar system formed (but long enough to have been included into meteoritic material), hence the assumption that it came from a supernova.

If the solar system did form near a supernova, the relative abundances of other isotopes, like those of oxygen, are expected to also have been altered from injection of supernova ejecta. Oxygen has three stable isotopes $-{ }^{16} \mathrm{O},{ }^{17} \mathrm{O}$, and ${ }^{18} \mathrm{O}$, the terrestrial (SMOW) abundance of which has been measured to be ${ }^{17} \mathrm{O} /{ }^{16} \mathrm{O}=3.8288 \cdot 10^{-4}$ and ${ }^{18} \mathrm{O} /{ }^{16} \mathrm{O}=2.0052 \cdot 10^{-3}$. Thus, knowing the post-injection oxygen isotopic composition and the amount of oxygen isotopes injected from the supernova, the pre-injection oxygen isotopic composition of the solar system can be predicted (Gounelle \& Meibom 2007). This can serve as another test for the supernova injection model.

Defining the post-injection composition of the solar system is tricky. A good first start is to assume that the SMOW composition is approximately the post-injection composition. However, there are complicated processes in the solar system (while it was forming and also today) that can alter the relative abundances of the oxygen isotopes, e.g. CO-self shielding (e.g. Lyons \& Young 2005). The next step towards improvement that one can make is to find solar system material that is known to have formed closer (in time) to the injection of supernova material. The oldest known solids that formed in the solar system are CAIs - Calcium- and Aluminum- rich inclusions (Amelin et al. 2002) -, which presumably formed after the contribution of supernova ejecta. The reason they are believed to have formed after the injection is that they contain the short-lived radionuclides for whose origin the supenova injection model has been invoked. The oxygen isotopic composition of CAIs is $\delta^{17} \mathrm{O}=-40 \%{ }^{0}$ and $\delta^{18} \mathrm{O}=-40^{0} / 00$ (in the delta-notation, the abundances are given relative to SMOW abundances in permille). Thus we assume that this is an appropriate post-injection oxygen isotopic composition.

The initial abundance of ${ }^{26} \mathrm{Al}$ in the Early Solar System has been very well determined as ${ }^{26} \mathrm{Al} /{ }^{27} \mathrm{Al}=$ $5 \cdot 10^{-5}$ (MacPherson et al. 1995). This abundance is used to determine the fraction of the yield that was added to the solar system inventory. Using simple mass balance, the pre-injection $\mathrm{O}$ isotopic composition was calculated. Table 1 lists our results, and is explained in the next paragraph.

\section{Method and Results}

Similar to Gounelle \& Meibom (2007), we consider two different scenarios - the maximum and the minimum impact scenario. The maximum impact scenario assumes that all of the $\mathrm{O}$ isotopes in the considered fraction is injected. The minimum impact scenario considers only those $\mathrm{O}$ isotopes that are bound up into dust (with $\mathrm{Mg}, \mathrm{Al}, \mathrm{C}, \mathrm{Ca}, \mathrm{Si}, \mathrm{Ti}, \mathrm{Fe}$ ).

For each scenario we consider a few different cases. The first case takes into account the entire ejecta of the simulation (column Bulk in table Results). Because of fragmentation of the ejecta into high density knots it makes sense to also consider isolated regions of the ejecta. The ${ }^{26} \mathrm{Al}$-rich regions together contain about $92 \%$ of all the ${ }^{26} \mathrm{Al}$ in the supernova, but only $\sim 5 \%$ of the ${ }^{16} \mathrm{O}$ and $\sim 10 \%$ each of ${ }^{17} \mathrm{O}$ and ${ }^{18} \mathrm{O}$. Therefore we deemed it necessary to look at the ${ }^{26} \mathrm{Al}$-rich regions individually. We calculated the pre-injection $\mathrm{O}$ isotopic composition for the following different cases: injection from the Bubble (column Bubble), the Ring (column Ring), an ${ }^{18} \mathrm{O}$-rich region of 
the Torus (column Torus), and the region immediately outside the Bubble (column Outside bubble). The Torus region and the region outside the Bubble (the Outside region) contain very little ${ }^{26} \mathrm{Al}$, however, they might be of interest. The Outside region is adjacent to the Bubble - the most promising region- and perhaps some of the Outside might mix with the Bubble. The Torus is ${ }^{18} \mathrm{O}$-rich.

We selected the ${ }^{26} \mathrm{Al}$-rich regions based on the ${ }^{26} \mathrm{Al}$ content. We set the threshold for inclusion into the ring to $\mathrm{M}\left({ }^{26} \mathrm{Al}\right)>1.5 \cdot 10^{-13} \mathrm{M}_{\odot} /$ particle, and to $\mathrm{M}\left({ }^{26} \mathrm{Al}\right)>1.5 \cdot 10^{-11} \mathrm{M}_{\odot} /$ particle for inclusion into the Bubble. For comparison, the amount of ${ }^{26} \mathrm{Al}$ needed is $\sim 10^{-10} \mathrm{M}_{\odot}$. The Torus and the Outside region were selected based on location.

\section{Conclusion}

First results from the Genesis Mission indicate that the solar oxygen abundance relative to SMOW is $\delta^{17} \mathrm{O} \sim-60^{0} / 00$ and $\delta^{18} \mathrm{O} \sim-60^{0} / 00$. Comparing this to our inferred pre-injection delta-values leads to a few different conclusion. First, our inferred pre-injection $\delta^{17} \mathrm{O}$ values were shown to match the solar value reasonably well. The Bubble and the bulk ejecta (minimum impact scenario) are the most promising regions. Other regions and scenarios show larger shift in $\delta^{17} \mathrm{O}$, which could, in theory, be reduced by decreasing the fraction of oxygen injected (e.g. if amount of $\mathrm{O}$ in dust is overestimated).

Second, the shifts in the oxygen delta-values need not necessarily be large, as is evident in the Bubble and the Outside (minimum impact scenario). Since a number of the particles in the Bubble already contain more than the required amount of ${ }^{26} \mathrm{Al}$, the standard threshold $\left(1.5 \cdot 10^{-13} \mathrm{M}_{\odot}\right.$ of ${ }^{26} \mathrm{Al}$ ) cases could be more of a worst- case-scenario. Using a more moderate threshold of 1.5 . $10^{-11} \mathrm{M}_{\odot}$ decreases the extend of the ${ }^{26} \mathrm{Al}$ rich regions and results in the injected $\mathrm{O}$ having a much smaller impact.

Third, all cases show that there seems to be not enough ${ }^{18} \mathrm{O}$ to give a shift towards the solar $\delta^{18} \mathrm{O}$. This is unexpected and a little puzzling at the moment. It is possible that some ${ }^{18} \mathrm{O}$ produced by decay have not yet been properly included.

\section{References}

[1] Amelin, Y., Krot, A. N., Hutcheon, I. D., \& Ulyanov, A. A. 2002, Science, 297, 1678

[2] Desch, S. J., \& Ouellette, N. 2005, 36th Annual Lunar and Planetary Science Conference, 36, 1327

[3] Fryer, C. L., Rockefeller, G., \& Warren, M. S. 2006, ApJL, 643, 292

[4] Gounelle, M., \& Meibom, A. 2007, ApJL, 664, L123

[5] Lyons, J. R., \& Young, E. D. 2005, Nature, 435, 31

[6] MacPherson, G. J., Davis, A. M., \& Zinner, E. K. 1995, Meteoritics, 30, 365

[7] Tachibana, S., Huss, G. R., Kita, N. T., Shimoda, H., \& Morishita, Y. 2005, 36th Annual Lunar and Planetary Science Conference, 36, 1529

[8] Young, P. A., et al. 2006, ApJ, 640, 891

[9] Young, P. A., \& Arnett, D. 2005, ApJ, 618, 908

[10] Young, P. A., \& Fryer, C. L. 2007, ApJ, 664, 1033 